\title{
Editorial
}

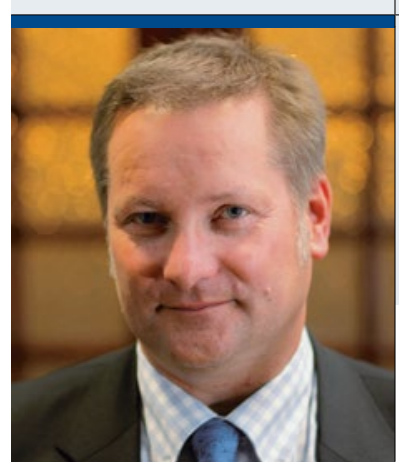

"Die Sonografie ist das Stethoskop des Urologen."

Prof. Dr. med. Tillmann Loch

Klinik für Urologie, Diakonissenkrankenhaus Flensburg, Lehrkrankenhaus der

Christian-Albrechts-Universität Kiel, Flensburg

\section{Bildgebung: Haben Sie Mut, zeigen Sie Ihre Fähigkeiten!}

D ie bildgebende Diagnostik in der Früherkennung und in der Verlaufsbeobachtung von urologischen Erkrankungen stellt ein Herzstück der Urologie dar. So wird sowohl im Krankenhaus als auch in der Praxis nach Anamnese und Laboruntersuchungen fast immer auch eine sonografische Grunduntersuchung durchgeführt.

Die Möglichkeiten der urologischen Bildgebung reichen von perkutaner, abdominell/retroperitonealer Sonografie über Endosonografie und perinealer Sonografie bis hin zu Leeraufnahmen, intravenösen Urogrammen und Durchleuchtung. Seit den 1980erJahren hat sich vor allem der transrektale Ultraschall (TRUS) zu einem Standardwerkzeug in der Urologie entwickelt. Neben dem konventionellen Ultraschall gehört auch die interventionelle Bildgebung zur täglichen Arbeit eines Urologen, zum Beispiel bei perkutanen Entlastungen von Harnstauungsnieren oder bei gezielten Prostatastanzbiopsien.

Die Bildgebung in der Urologie ermöglicht dem Behandler Pathologika, Normalbefunde und abnormale Erscheinungen Symptomkomplexen direkt zuzuordnen. Dadurch können Erkrankungen komplex und komplett beurteilt sowie Therapieoptionen, Komplikationen und Erfolgsaussichten aus eigener Erfahrung und Hand eingeschätzt werden. Erst dies erlaubt eine exakte Risikoeinschätzung der spezifischen Konstellation und damit eine Beratung des Patienten im Sinne einer individualisierten Medizin.

\section{Integrierte Versorgungslösungen sind urologisch möglich und sinnvoll}

An vielen Stellen hat sich eine exzellente urologische Bildgebung etabliert, die - wie beim TRUS der Prostata - fast ausschließlich in der Urologie eingesetzt und beherrscht wird. Dabei ist ein enormes Knowhow entstanden, das zum Beispiel bei der Biopsie der Prostata in sämtliche Leitlinien Eingang gefunden hat. Auch werden im Fach Urologie Neuentwicklun- gen geschaffen, die den Vergleich mit radiologischen Schnittbildtechniken nicht scheuen müssen, beispielsweise die Elastografie und die neue UltraschallComputertomografie (US-CT), mit der Möglichkeit longitudinaler Beobachtung. Mut zur urologischen Bildgebung lohnt sich, denn oft lassen sich in Zusammenhang mit den von uns Urologen erhobenen klinischen Befunden bereits exzellente Aussagen treffen.

Zur Ausbildung zum Urologen gehört unbedingt eine strukturierte Schulung im Umgang mit der Bildgebung. Gemeinsam mit der Sektion Urologie der Deutschen Gesellschaft für Ultraschall in der Medizin (DEGUM) bietet der Arbeitskreis Bildgebende Sektion der Akademie der Deutschen Urologen eine breit gefächerte Ausbildung an. Sie erstreckt sich von Workshops und Kursen auf Regionaltagungen über Kurse auf Jahrestagungen bis hin zu Anwenderseminaren mit Live-Untersuchungen.

In dieser Ausgabe der URO-NEWS werden Basis-, Spezial- und Schnittbildtechniken, die auch vom Radiologen durchgeführt werden, vorgestellt und dabei Kenntnisse und Know-how aus der Praxis für die Praxis vermittelt.

Die Autoren der Fortbildungsbeiträge haben ein breites Wissen und Kenntnis in praktischer sowie theoretischer Durchführung der bildgebenden Methoden und sind auf der Höhe der maßgeblichen Literatur im Bereich der Bildgebung, sodass sowohl die Leser aus der Klinik als auch diejenigen aus der Praxis profitieren.

Zeigen auch Sie sich und Ihre Fähigkeiten in der urologischen Bildgebung im Sinne der Patienten!

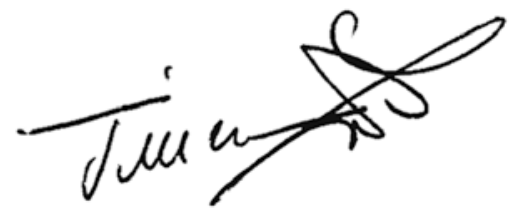

